\title{
Boundary integral for the Ramachandran index
}

\author{
Paolo Antonini (*)
}

ABstRACT - The computation for the Ramachandran index for Galois coverings and foliations is reduced to a solely boundary computation. This is a reminescence of the classical theory.

Mathematics SubJect Classification (2012). 19K56, 58J32.

KEYworDs. Index theory, APS boundary value problems, coverings, foliations.

\section{Introduction}

Let $X$ be a compact connected Riemannian manifold with boundary $Y$ and product structure near the boundary. Denote by $D$ a compatible Dirac operator on $X$, and by $R$ a pseudo differential boundary condition. One of the main results of the classical theory is that the $L^{2}$-realization $D_{R}$ of the Dirac operator $D$ with boundary condition $R$ is Fredholm and its index is equal to the Fredholm index of an operator which lives solely on the boundary. This is called the boundary integral:

$$
R \mathcal{P}_{+}: H_{+} \longrightarrow \text { Range }(R),
$$

where $H_{+}$is the space of Cauchy datas, i.e. the traces of smooth solutions on the boundary and $\mathcal{P}_{+}$is a certain pseudo differential projection on $H_{+}$, called the Calderon projection. The importance of the Calderon projection in the theory of boundary value problems can hardly be over-emphasized: we refer the reader to the excellent monograph [4]. In particular, this applies to the case of the celebrated Atiyah-Patodi-Singer boundary condition. In this case the index of $D_{R}$ is called the APS index.

(*) Indirizzo dell'A.: Dipartimento di Matematica, Università "La Sapienza", P.le Aldo Moro 5, 00185 Roma.

E-mail: paolo.anton@gmail.com 
The above result can be informally summarized as the following motto: "The APS index is a boundary computation.", and so immediately suggests the following question: "Does the Ramachandran APS index for Galois coverings and foliations also reduce to a boundary computation?".

By an adaptation of the argument in [11], we show that the answer to this question is yes. More precisely, given a Galois covering $X \rightarrow X=X / \Gamma$, we exhibit two subspaces of sections on the boundary that are essentially isomorphic as Hilbert $\Gamma$-modules to (and so in particular have the same $\Gamma$ dimensions of) the kernel and the cokernel of the Dirac operator as considered by Ramachandran. For $\Gamma$ the trivial group we recover that the APS index can be expressed by a boundary computation. However, it should be remarked that in the classical case, the known result on the APS index are much more refined than what we are recovering here. Namely, as remarked above, in the classical case, the boundary computation of the APS index is expressed in terms of a pseudodifferential operator, whereas in our approach no such description is available.

\section{The Dirac operator and geometrical setting}

We adopt the same geometric setting as Ramachandran [11]; a comprehensive reference for the theory of Dirac operators we will need in the present paper is the book by John Roe [12]. So let $X$ be a compact manifold with boundary $\partial X=Y$ and $(D, S, \varepsilon)$ a geometric Dirac operator acting on the sections of a graded Clifford bundle $S$ with grading operator $\varepsilon$. For instance $D$ can be the Dirac operator on a Clifford module $S$ on an even dimensional manifold, endowed with the canonical $Z_{2}$-grading. In particular $S$ is a $Z_{2}$ graded bundle of Clifford modules on the bundle of Clifford algebras associated to a Riemannian metric equipped with compatible Hermitian structure and connection. This means that

- the Clifford action of vector fields on the module $C^{\infty}(X, S)$ of smooth sections of $S$ is odd (goes from $C^{\infty}\left(X, S^{+}\right)$to $C^{\infty}\left(X, S^{-}\right)$and viceversa) and skew-adjoint, i.e., $\left\langle v \cdot s_{1} \mid s_{2}\right\rangle+\left\langle s_{1} \mid v \cdot s_{2}\right\rangle=0$ for any vector field $v$ and sections $s_{1}$ and $s_{2}$ of $S$;

- The connection $\nabla^{S}$ on $S$ is compatible with the Clifford multiplication and with Levi Civita connection i.e. it is a derivation of $C^{\infty}(X, S)$ as a Clifford module over $C^{\infty}(X, T X)$ :

$$
\nabla_{v}^{S}(w \cdot s)=\left(\nabla_{v}^{\mathrm{LC}} w\right) s+w \cdot \nabla_{v}^{S_{s}}
$$

for any vector fields $v, w$ and any section $s$ of $S$. In the following we shall suppress the superscripts $S$ and LC. 
The resulting Dirac operator is odd

$$
D^{ \pm}: C^{\infty}\left(X, S^{ \pm}\right) \longrightarrow C^{\infty}\left(X, S^{\mp}\right) .
$$

We assume that all the geometric structure is product type near the boundary. This means that there exist an open neighborhood $Y \times[0,1)$ of $Y$ in $X$, with local coordinates $(y, u)$, on which:

- the Riemannian metric has the form $g_{i j}(y) d y^{i} \otimes d y^{j}+d u \otimes d u$;

- the Dirac operator has the special form

$$
\sigma \circ\left(\partial_{u} \otimes \mathrm{Id}+\mathrm{Id} \otimes Q\right)
$$

where $\sigma=\operatorname{Cl}\left(\partial_{u}\right)$ is the Clifford multiplication by the vector field $\partial_{u}$ and $Q$ is an essentially selfadjoint Dirac operator on $C^{\infty}(Y, S)$ commuting with $\sigma$ ( $Q$ is even).

By abuse of notation, we will simply write $D=\sigma\left(\partial_{u}+Q\right)$ to denote the boundary behavior of the Dirac operator.

Since the Clifford multiplication acts pointwise on the sections of $S$, the operator $\sigma$ induces a morphism

$$
\sigma: C^{\infty}\left(Y, S^{ \pm}\right) \rightarrow C^{\infty}\left(X, S^{\mp}\right)
$$

between the sections of the restriction of $S$ to $Y$. If we denote by

$$
b: C^{\infty}\left(X, S^{ \pm}\right) \rightarrow C^{\infty}\left(Y, S^{ \pm}\right)
$$

the restriction to the boundary, then we have the following Green-type formula with respect to the $L^{2}$-product on compactly supported sections which are smooth up to the boundary:

$$
\left\langle\left\langle s_{1} \mid D s_{2}\right\rangle\right\rangle_{X}-\left\langle\left\langle D s_{1} \mid s_{2}\right\rangle\right\rangle_{X}=\int_{Y}\left\langle\sigma b s_{1}, b s_{2}\right\rangle d \operatorname{vol}_{Y},
$$

where $\langle\langle-\mid-\rangle\rangle_{X}$ is the $L^{2}$-inner product on sections of $S$,

$$
\left\langle\left\langle s_{1} \mid s_{2}\right\rangle\right\rangle_{X}=\int_{X}\left\langle s_{1} \mid s_{2}\right\rangle d \operatorname{vol}_{X} .
$$

\section{The BGK expansion}

If $Y$ is a manifold with bounded geometry:

- completeness

- positive injectivity radius

- uniformly bounded curvature tensor (with all the covariant derivatives) 
one is allowed to use the so called BGK expansion (after Berezanskii, Browder, Garding, Gel'fand and Kac), i.e. the existence of a generalized eigensection expansion for the elliptic selfadjoint differential operator $Q$. More precisely, there exists a sequence of measures $\mu_{j}$ on $\mathbb{R}$ and measurable maps

$$
e_{j}: \mathbb{R} \times Y \longrightarrow S
$$

such that $e_{j}(\lambda, \cdot)$ is a smooth section of $S$ over $Y$ (for almost every $\lambda$, with respect to the meaure $\mu_{j}$ ) with

$$
Q e_{j}(\lambda, \cdot)=\lambda e_{j}(\lambda, \cdot) .
$$

Furthermore for a section $s \in C_{c}^{\infty}\left(Y, S_{\mid Y}\right)$ one has the sequence of functions on $\mathbb{R}$,

$$
(V s)_{j}(\lambda):=\left\langle\left\langle s \mid e_{j}(\lambda, \cdot)\right\rangle\right\rangle_{Y}=\int_{Y}\left\langle s \mid e_{j}(\lambda, \cdot)\right\rangle d \operatorname{vol}_{Y} .
$$

Each of these functions is square integrable with respect to the measure $\mu_{j}$, i.e., $(V s)_{j} \in L^{2}\left(\mathbb{R}, \mu_{j}\right)$ and so $V$ defines a linear operator $C_{c}^{\infty}\left(Y, S_{\mid Y}\right) \rightarrow$ $\oplus_{j} L^{2}\left(\mathbb{R}, \mu_{j}\right)$ which is seen to extend to an isometry of Hilbert spaces

$$
V: L^{2}\left(Y, S_{\mid Y}\right) \longrightarrow \bigoplus_{j} L^{2}\left(\mathbb{R}, \mu_{j}\right)
$$

i.e., one has

$$
\int_{Y}|s|^{2} d \operatorname{vol}_{Y}=\sum_{j} \int_{\mathbb{R}}\left|(V s)_{j}\right|^{2} d \mu_{j} .
$$

Furthermore $V$ intertwines the Borel functional calculus of $Q$ with the action of jointly (i.e., for any $j$ ) Borel measurable functions by multiplication on $L^{2}\left(\mathbb{R}, \mu_{j}\right)$, i.e., one has

$$
(V f(Q) s)_{j}=f(\lambda)(V s)_{j}
$$

for any $j$ and

$$
\operatorname{Domain}(f(Q))=\left\{s:\left.\sum_{j} \int_{\mathbb{R}}|f(\lambda)|^{2}(V s)_{j}(\lambda)\right|^{2} d \mu_{j}(\lambda)<\infty\right\} .
$$

\section{Sobolev spaces of sections}

It is customary to define a scale $W^{k}(X, S)$ of Sobolev spaces of sections by means of the Dirac operator $D$ as follows: for any nonnegative integer $k$, the Sobolev space $W^{k}(X, S)$ is defined to be the completion of $C_{c}^{\infty}(X, S)$ with 
respect to the Sobolev norm

$$
\|s\|_{k}:=\left(\|s\|_{2}^{2}+\|D s\|_{2}^{2}+\cdots\left\|D^{k} s\right\|_{2}^{2}\right)^{1 / 2}
$$

where $\|\cdot\|_{2}$ is the $L^{2}$-norm on $C_{c}^{\infty}(X, S)$. Note that the support of a section in $C_{c}^{\infty}(X, S)$ may have a nonempty intersection with the boundary $Y$ of $X$. Negative order Sobolev spaces are defined by duality and are considered as spaces of distributional sections.

One also considers the topological vector space

$$
W^{\infty}(X, S)=\bigcap_{k \geq 0} W^{k}(X, S),
$$

endowed with its canonical Fréchet topology, and

$$
W^{-\infty}(X, S)=\bigcup_{k \leq 0} W^{k}(X, S)
$$

with the weak-* topology induced by the duality with $W^{\infty}(X, S)$. It can be shown (see, e.g., [12]) that a continuous linear operator from $W^{-\infty}(X, S)$ to $W^{\infty}(X, S)$ is represented by a smoothing kernel which is smooth up to the corners on $X \times X$. Moreover both the kernel and all their covariant derivatives are uniformly bounded.

In an analogous way, one defines a scale of boundary Sobolev spaces by means of the essentially selfadjoint operator $Q$ on $Y$. Namely, for a non negative integer $k$ one defines $W^{k}\left(Y, S_{\mid Y}\right)$ as the closure of $C_{c}^{\infty}(Y, S)$ under the norm

$$
\left(\|s\|+\|Q s\|^{2}+\cdots\left\|Q^{k} s\right\|^{2}\right)^{1 / 2}
$$

One has

$$
W^{k}(Y, S)=\operatorname{Domain}\left(\left(Q^{*} Q\right)^{k / 2}: L^{2}\left(Y, S_{\mid Y}\right) \rightarrow L^{2}\left(Y, S_{\mid Y}\right)\right),
$$

so that one can use this identity to define fractional Sobolev spaces $W^{k}(Y, S)$ with a real positive $k$. By means of the BGK expansion one sees that the trace operator $b: C_{c}^{\infty}(X, S) \longrightarrow C_{c}^{\infty}\left(Y, S_{\mid Y}\right)$ extends to a well defined bounded operator

$$
W^{k}(X, S) \longrightarrow W^{k-1 / 2}\left(Y, S_{\mid Y}\right), \quad k \in \mathbb{N} .
$$

Analogously, by using $D^{ \pm}$in place of $D$, one defines the spaces of sections $W^{k}\left(X, S^{ \pm}\right)$and $W^{k}\left(Y, S^{ \pm}\right)$. 


\section{The APS boundary condition}

We now relax the compactness assumption on $X$ by requiring instead that $X$ is a cocompact Galois cover with deck transformation group $\Gamma$. All the bundles and operators from the previous section will therefore be assumed to be $\Gamma$-equivariant, while the measure on $X$ will be assumed to be $\Gamma$-invariant.

Following an idea of Roe, Ramachandran introduces the crucial concept of selfadjoint boundary condition. It is a $\Gamma$-equivariant operator

$$
B: C_{c}^{\infty}(Y, S) \longrightarrow C^{\infty}(Y, S) \cap L^{2}(Y, S)
$$

such that

- $B$ extends to a selfadjoint bounded operator on $L^{2}(Y, S)$

- $\sigma B+B \sigma=\sigma$

- if $s_{1}, s_{2} \in C_{c}^{\infty}(X, S)$ are such that $B b s_{i}=0$ then $\left\langle\left\langle D s_{1}, s_{2}\right\rangle\right\rangle_{X}=$ $\left\langle\left\langle s_{1}, D s_{2}\right\rangle\right\rangle_{X}$.

In the above formulas, $\sigma$ is the Clifford multiplication by the unit normal vector to the boundary $Y$ and $b$ is the restriction to the boundary (see section 2 ).

It is no surprise that the prototypical selfadjoint boundary condition is the Atiyah-Patodi-Singer operator.

Let us recall its definition. The operator $\sigma$ of Clifford multiplication by the unit normal vector to the boundary $Y$ satisfies $\sigma \sigma^{*}=1$ and $\sigma^{2}=-1$. Being odd, this gives

$$
\sigma=\left(\begin{array}{cc}
0 & -\sigma_{+}^{*} \\
\sigma_{+} & 0
\end{array}\right)
$$

The even operator

$$
Q=\left(\begin{array}{cc}
Q_{+} & 0 \\
0 & Q_{-}
\end{array}\right)
$$

commutes with $\sigma$, so that the operator

$$
B_{\mathrm{APS}}=\left(\begin{array}{cc}
B_{\mathrm{APS}}^{+} & 0 \\
0 & B_{\mathrm{APS}}^{-}
\end{array}\right)=\left(\begin{array}{cc}
\chi_{[0,+\infty)}\left(Q_{+}\right) & 0 \\
0 & \chi_{(0,+\infty)}\left(Q_{-}\right)
\end{array}\right),
$$

where $\chi_{A}$ is the characteristic function of a Borel measurable subset $A$ of $\mathbb{R}$, satisfies the equation $\sigma B_{\mathrm{APS}}+B_{\mathrm{APS}} \sigma=\sigma$. One easily checks that $B_{\mathrm{APS}}$ 
satisfies the other two properties characterizing selfadjoint boundary conditions. Also, by the equations $\sigma B_{\mathrm{APS}}+B_{\mathrm{APS}} \sigma=\sigma$ and $\sigma \sigma^{*}=1$, we get $\sigma B_{\mathrm{APS}} \sigma^{*}=1-B_{\mathrm{APS}}$, i.e., the relation

$$
1-\sigma_{+} B_{\mathrm{APS}}^{+} \sigma_{+}^{*}=B_{\mathrm{APS}}^{-},
$$

which will play a crucial role in our proof of the boundary behavior of Ramachandran $\Gamma$-index.

For $k$ a positive integer, let $W_{\mathrm{APS}}^{k}(X, S) \subseteq W^{k}(X, S)$ be the subspace of sections satisfying the A.P.S. boundary condition:

$$
W^{k}(X, S)_{\mathrm{APS}}:=\left\{s \in W^{k}(X, S): B_{\mathrm{APS}} b s=0\right\} .
$$

Ramachandran proves that $D$ acting on $W_{\mathrm{APS}}^{\infty}(X, S)$ is essentially selfadjoint.

This follows and idea of John Roe and is builded on the existence of compatible bounded operators $R_{1}^{(k)}$ and $R_{2}^{(k)}$

$$
R_{i}^{(k)}: W^{k}(X, S) \longrightarrow W_{\mathrm{APS}}^{k+1}(X, S), \quad k \in \mathbb{N}
$$

such that

$$
D R_{1}^{(k)}-1=\rho_{1}^{(k)}, \quad R_{2}^{(k)} D-1=\rho_{2}^{(k)}
$$

where the $\rho_{i}^{(k)}$ are smoothing operators. To be precise, to say that $D$ acting on $W_{\mathrm{APS}}^{\infty}(X, S)$ is essentially selfadjoint means that its minimal domain is $W_{\mathrm{APS}}^{1}(X, S)$ and that on this domain the operator $D$ coincides with its adjoint as unbounded operator on a Hilbert space. The operators $R_{i}^{(k)}$ are shown to exist by a standard technique of gluing an interior parametric with a boundary one. The regularizing properties of this parametrix also imply that the domain of $D^{k}$ is contained in $W^{k}(X, S)$. Moreover, by the spectral theorem, if $f$ is a rapidly decreasing Borel function on $\mathbb{R}$, then

$$
f(D): W^{-k_{1}}(X, S) \longrightarrow W^{k_{2}}(X, S)
$$

for every pair of nonnegative integers $k_{1}$ and $k_{2}$ and therefore $f(D)$ is represented by a smooth kernel. There is probably no need to recall that the fundamental example of this phenomenon is the smoothing operator given by the heat operator $e^{-t D^{2}}$, playing a crucial role in the classical APS formula.

By building a parametrix for the initial boundary value problem on the cylinder $[0, \infty) \times Y$, Ramachandran is able to exhibit a 1-parameter family of operators $E(t)$ mapping $W^{-k_{1}}(X, S)$ into $W^{k_{2}}(X, S)$ for every couple of nonnegative integers $k_{1}$ and $k_{2}$, and such that for $k_{1}, k_{2}>0$ the difference

$$
e^{-t D^{2}}-E(t): W^{-k_{1}}(X, S) \longrightarrow W^{k_{2}}(X, S)
$$


satisfies

$$
\left\|e^{-t D^{2}}-E(t)\right\| \leq C t^{\alpha}, \quad 0 \leq t \leq 1,
$$

for some $C, \alpha>0$. Here the norm on the left is the operator norm in the space of bounded operators between $W^{-k_{1}}(X, S)$ and $W^{k_{2}}(X, S)$. This result is a direct consequence of the Duhamel principle.

Finally, note that since the APS boundary condition is an even operator, it is meaningful to consider the spaces of boundary conditions $W^{k}\left(X, S^{ \pm}\right)$APS defined by

$$
W_{\mathrm{APS}}^{k}=\left\{s \in W^{p}\left(X, S^{ \pm}\right): B_{\mathrm{APS}}^{ \pm} b s=0\right\} .
$$

Also, one considers the $L^{2}$-realization of the operator $D^{ \pm}$with boundary condition $B_{\mathrm{APS}}^{ \pm}$. They are the operators

$$
D_{\chi_{[0,+\infty)}^{+}\left(Q_{+}\right)}: W^{1}\left(X, S^{+}\right)_{\mathrm{APS}} \longrightarrow L^{2}\left(X, S^{-}\right)
$$

and

$$
D_{\chi_{(0,+\infty)}^{-}\left(Q_{-}\right)}^{-}: W^{1}\left(X, S^{-}\right)_{\mathrm{APS}} \longrightarrow L^{2}\left(X, S^{+}\right)
$$

defined as the restrictions of $D^{ \pm}$to the subspaces $W^{1}\left(X, S^{ \pm}\right)_{\text {APS }}$.

The argument used by Ramachandran in [11, Theorem 4.1] shows that $D_{\chi_{[0,+\infty)}^{+}\left(Q_{+}\right)}$is closed, and that its Hilbert space adjoint is $D_{\chi_{(0,+\infty)}^{-}\left(Q_{-}\right)}^{-}$. More precisely, Ramachandran's argument shows that the adjoint of $D^{+}$is the operator $D^{-}$with the adjoint boundary condition $\sigma_{+}\left(1-B_{\mathrm{APS}}^{+}\right) \sigma_{+}^{*}$. But, by equation (5.1), this is precisely the boundary condition $B_{\mathrm{APS}}^{-}$.

\section{The finite dimensionality of the Breuer Fredholm index}

We recall the setting. Here $X \longrightarrow X / \Gamma$ is a regular covering of a compact manifold with boundary $X / \Gamma$. In particular the action of $\Gamma$ a discrete group is free and cocompact and the deck transformation group is exactly $\Gamma$. This is the same of $\Gamma$ principal bundle. Since $S$ is a $\Gamma$-equivariant bundle, we have a natural induced linear action of $\Gamma$ on the space of sections $L^{2}(X ; S)$. Denote by $\operatorname{End}_{\Gamma} L^{2}(X ; S)$ the normed algebra of all bounded operators on $L^{2}(X ; S)$ commuting with the $\Gamma$-action. It is a semifinite von Neumann algebra equipped with a semifinite trace $\operatorname{Tr}_{\Gamma}$. This is easily seen choosing a fundamental domain $\mathcal{D}$. The $L^{2}$ space of section decomposes as a Hilbert space tensor product $L^{2}(\mathcal{D}) \otimes l^{2}(\Gamma)$ with the $\Gamma$-action given by the left regular representation. Correspondingly one can identify the relevant von Neumann algebra with $B\left(L^{2}(\mathcal{D})\right) \otimes \operatorname{vn}(\Gamma)$ where $\operatorname{vn}(\Gamma)$ is the von 
Neumann algebra of the discrete group $\Gamma$ which is manifestly semifinite. The trace $\operatorname{tr}_{\Gamma}$ is given by the evaluation of the coefficient in the identity of $\Gamma$ in the representation of the elements of $l^{2}(\Gamma)$ as sequences

$$
\sum_{\gamma \in \Gamma} \alpha_{\gamma} \cdot \gamma, \quad \alpha_{\gamma} \in \mathbb{C} .
$$

It follows that $B\left(L^{2}(\mathcal{D})\right) \otimes \operatorname{vn}(\Gamma)$ is also semifinite with the trace identified with

$$
\operatorname{tr}_{B\left(L^{2}(\mathcal{D})\right.} \otimes \operatorname{tr}_{\Gamma}
$$

We shall need the notion of Breuer-Fredholm operator. So let $M$ be a Von Neumann agebra with a trace $\tau: M^{+} \longrightarrow[0, \infty]$ one has a natural notion of dimension of a closed subspace affiliated to $M$, i.e. a subspace $V$ whose projection $\operatorname{Pr}_{V}$ belongs to $M$. This is by definition the relative dimension $\tau\left(\operatorname{Pr}_{V}\right)$. Relative dimension is the cornerstone of a theory of Fredholm operators inside $M$. This story goes back to the seminal work of Breuer [5, $6]$. For this reason relatively Fredholm operators are called BreuerFredholm. A Breuer-Fredholm operator has a finite real index with some stability properties as in the classical theory. More precisely an operator $T$ affiliated to $M$ (this means that $T$ can also be unbounded but all its spectral projections belong to $M$ ) is called Breuer-Fredholm if the projection to the kernel of $T$ is finite relatively to $M$ and the range contains the range of a cofinite projection. All of this fits in the setting of Hilbert modules over a von Neumann algebra [7, 13]. In the specific setting of a von Neumann algebra of a discrete group these are pre-Hilbert spaces $H$ together with a unitary action of $\Gamma$. Furthermore there is a technical but simple condition of projectivity which is automatically satisfied in our situation. Using the von Neumann algebra of $\Gamma$ a suitable notion of (renormalized) real valued additive dimension of $\Gamma$-Hilbert modules is well defined. In particular if $H_{1}$ and $H_{2}$ are $\Gamma$-Hilbert modules that are essentially isomorphic i.e. there exists a bounded equivariant map $f: H_{1} \longrightarrow H_{2}$ with dense range they have the same dimension [13]. Indeed the unitary appearing in the polar decomposition of $f$ gives an isomorphism of Hilbert modules. We shall select a subalgebra $\mathcal{U}$ of $\operatorname{End}_{\Gamma} L^{2}(X ; S)$ where the trace is finite. We say that a bounded $\Gamma$-equivariant endomorphism $A$ of $L^{2}(X ; S)$ belongs to $\mathcal{U}$ if

- $A$ has a smooth integral kernel $k$, i.e., there exist a $\Gamma$-equivariant smooth section of the vector bundle $\operatorname{Hom}\left(\pi_{2}^{*} S, \pi_{1}^{*} S\right)$ on $X \times X$ such that

$$
A=\pi_{1 *} \circ k \circ \pi_{2}^{*},
$$


i.e., there exist $k(x, y) \in \operatorname{Hom}\left(S_{y}, S_{x}\right)$ smoothly depending on $x$ and $y$, such that $k(g x, g y)=g k(x, y) g^{-1}$ and

$$
(A s)(x)=\int_{X} k(x, y) s(y) d y, \quad s \in L^{2}(X, S) ;
$$

- $k$ is fibrewise uniformly bounded in the $L^{2}$-norm, i.e. there exists a constant $C \int_{X}\|k(x, y)\|^{2} d y<C$ and $\int_{X}\|k(x, y)\|^{2} d x<C$ for all $x, y \in X$;

- $k$ is smoothing, i.e., the maps $s_{y} \mapsto k(\cdot, y) s_{y}$ and $s_{x} \mapsto\left\langle s_{x} \mid k(x, \cdot)\right\rangle$ are smooth maps from the total space of the bundle $S$ to $L^{2}(X, S)$.

It is not difficult to show that $\mathcal{U}$ is an algebra, see [12, Lemma 15.2] and it is contained in the von Neumann algebra by definition because it is made of operators commuting with $\Gamma$. Since $k(x, x) \in \operatorname{End}\left(S_{x}\right)$, taking its trace we get a smooth real valued function on $X$; moreover, since $k$ is $\Gamma$-equivariant and the the trace of an endomorphism depends only on its conjugacy class, $x \mapsto \operatorname{trk}(x, x)$ descends to a smooth function on the compact manifold $X / \Gamma$. One then finds that the $\Gamma$-trace of an element $A$ of $\mathcal{U}$ is given by

$$
\operatorname{Tr}_{\Gamma}(A)=\int_{X / \Gamma} \operatorname{tr} k(x, x) d x .
$$

In particular, $\operatorname{Tr}_{\Gamma}(A)<+\infty$ for any $A$ in $\mathcal{U}$. Also notice that, if both $A_{1}$ and $A_{2}$ lie in the subalgebra $\mathcal{U}$, then

$$
\operatorname{Tr}_{\Gamma}\left(A_{1} A_{2}\right)=\operatorname{Tr}_{\Gamma}\left(A_{2} A_{1}\right) .
$$

One says that a closed subspace $H \subset L^{2}(X, S)$ is $\Gamma$-finite dimensional if the ortogonal projection on $H$ has finite $\Gamma$-trace. When this happens one defines

$$
\operatorname{dim}_{\Gamma}(H):=\operatorname{Tr}_{\Gamma}\left(\Pi_{H}\right) .
$$

In particular, $H$ is $\Gamma$-finite dimensional whenever $\Pi_{\Gamma}$ lies in $\mathcal{U}$. A remarkable instance of this is the following: since the Dirac operator $D$ is $\Gamma$ equivariant, for every rapidly decreasing Borel function $f$ on $\mathbb{R}$ the operator $f(D)$ is an element of $\mathcal{U}$, and so in particular the projections on the kernels of $D^{+}$and $D^{-}$are in $\mathcal{U}$. It follows that the $\Gamma$-index of $D$ is finite:

$$
\operatorname{ind}_{\Gamma}(D):=\operatorname{dim}_{\Gamma}\left(\operatorname{Ker} D^{+}\right)-\operatorname{dim}_{\Gamma}\left(\operatorname{Ker} D^{-}\right)<+\infty .
$$

Since $S$ is $Z_{2}$-graded, so are the spaces of sections $L^{2}(X, S)$ and the algebra of operators $\operatorname{End}_{\Gamma} L^{2}(X ; S)$, and the notion of $\Gamma$-trace immediately gen- 
eralizes to the notion of $\Gamma$-supertrace. Denoting by $\varepsilon: L^{2}(X ; S) \rightarrow L^{2}(X ; S)$ the $Z_{2}$-grading operator, the operator $\varepsilon e^{-t D^{2}}$ interpolates between $\varepsilon$ and the operator

$$
\left(\begin{array}{cc}
\Pi_{\mathrm{Ker}\left(D^{+}\right)} & 0 \\
0 & \Pi_{\mathrm{Ker}\left(D^{-}\right)}
\end{array}\right)
$$

Moreover, the $\Gamma$-supertrace of $\varepsilon e^{-t D^{2}}$ is independent of $t>0$, and one finds the McKean-Singer formula

$$
\operatorname{ind}_{\Gamma}(D)=\operatorname{Str}_{\Gamma}\left(\varepsilon e^{-t D^{2}}\right), \quad \forall t>0 .
$$

By computing the limit for $t \rightarrow 0$ of the right hand side, Ramachandran finds

$$
\operatorname{ind}_{\Gamma}(D)=\int_{X} \operatorname{ch}\left(\sigma_{D}\right) \operatorname{Td}(X)-\frac{\eta_{\Gamma}(0)+\operatorname{dim}_{\Gamma} \operatorname{Ker}\left(Q_{+}\right)}{2}
$$

where $\sigma_{D}$ is the principal symbol of the Dirac operator and

$$
\eta_{\Gamma}(0):=\frac{1}{\sqrt{\pi}} \int_{0}^{\infty} \operatorname{Tr}_{\Gamma}\left(Q_{+} e^{-t Q_{+}^{2}}\right) d t
$$

is the Ramachandran $\Gamma$-eta invariant. Notice that if $\Gamma$ is the trivial group, the Ramachandran $\Gamma$-index formula reduces to the classical APS index formula.

\section{The boundary integral}

To investigate the boundary behavior of the Dirac operator $D$ it is convenient to recall that on a collar $Y \times[0,1)$ the Clifford bundle $S$ is equipped with an isomorphism to the pullback bundle of the restriction $\left.S\right|_{Y}$ along the projection $Y \times[0,1) \rightarrow Y$. Via this isomorphism, the unit normal vector field $\partial_{u}$ on the boundary and the Clifford multiplication $\sigma=\operatorname{Cl}\left(\partial_{u}\right)$ act on the smooth sections of $S$ on the collar. Moreover, the action of $\partial_{u}$ and $\sigma$ on $C^{\infty}(Y \times[0,1), S)$ commute, since

$$
\nabla_{\partial_{u}}^{S}(\sigma s)=\nabla_{\partial_{u}}^{S}\left(\partial_{u} \cdot s\right)=\nabla_{\partial_{u}}^{\mathrm{LC}}\left(\partial_{u}\right) \cdot s+\partial_{u} \cdot \nabla_{\partial_{u}}^{S}(s)=\sigma \nabla_{\partial_{u}}^{S}(s) .
$$

In the above equation, the fact that the metric on the collar has the form $g_{i j}(y) d y^{i} \otimes d y^{j}+d u \otimes d u$ has been used to get $\nabla_{\partial_{u}}^{\mathrm{LC}}\left(\partial_{u}\right)=0$. Also, the 
Clifford multiplication $\sigma$ gives isomorphism

$$
\sigma_{ \pm}:\left.\left.S^{ \pm}\right|_{Y} \longrightarrow S^{\mp}\right|_{Y}
$$

thus, we have induced isomorphisms $\operatorname{Id} \oplus \sigma_{+}:\left.\left.\left.S^{+}\right|_{Y} \oplus S^{+}\right|_{Y} \rightarrow S\right|_{Y}$ and $\operatorname{Id} \oplus \sigma_{-}:\left.\left.\left.\left.S\right|_{Y} \rightarrow S^{+}\right|_{Y} \oplus S^{+}\right|_{Y} \rightarrow S\right|_{Y}$. With these isomorphisms in mind, the Dirac operator near the boundary can be rewritten as

$$
\begin{aligned}
\left(\operatorname{Id} \oplus \sigma_{-}\right) D\left(\operatorname{Id} \oplus \sigma_{+}\right): C^{\infty}\left(Y \times[0,1),\left.\left.S^{+}\right|_{Y} \oplus S^{+}\right|_{Y}\right) \longrightarrow & \\
& C^{\infty}\left(Y \times[0,1),\left.\left.S^{+}\right|_{Y} \oplus S^{+}\right|_{Y}\right) .
\end{aligned}
$$

Since $D=\sigma\left(\partial_{u}+Q\right)$ on the collar, this is precisely the operator

$$
\left(\begin{array}{cc}
0 & -\partial_{u}+Q_{+} \\
\partial_{u}+Q_{+} & 0
\end{array}\right),
$$

while the boundary condition $B_{\mathrm{APS}}$ is turned into the boundary condition

$$
\left(\begin{array}{cc}
\chi_{[0, \infty)}\left(Q_{+}\right) & 0 \\
0 & 1-\chi_{[0, \infty)}\left(Q_{+}\right)
\end{array}\right)
$$

on $\left.\left.S^{+}\right|_{Y} \oplus S^{+}\right|_{Y}$. In the derivation of (7.1) and (7.2), the commutation relation $Q \sigma=\sigma Q$ and the identity $\sigma^{2}=-1$ have been used.

Now let $\mathcal{H}^{+}$be the closed $\Gamma$-sub-Hilbert module of $L^{2}\left(Y, S^{+}\right)$defined as the range of

$$
\chi_{(-\infty, 0)}\left(Q_{+}\right): L^{2}\left(Y, S^{+}\right) \rightarrow L^{2}\left(Y, S^{+}\right),
$$

and define a map

$$
\gamma^{+}: \operatorname{Ker}\left(D_{\chi_{[0,+\infty)}^{+}\left(Q_{+}\right)}\right) \longrightarrow \mathcal{H}^{+}
$$

simply by restriction to the boundary. By the well known unique continuation property of the Dirac operator [4], a solution $\xi$ of the equation $D^{+} \xi=0$ that vanishes on the boundary (ghost solution) must vanish on the whole of $X$; thus restriction to the boundary gives a continuos injection of $\operatorname{Ker}\left(D_{\left.\chi_{[0,+\infty}\right)}^{+}\left(Q_{+}\right)\right.$into $\mathcal{H}$.

By the spectral Browder-Garding resolution one can represent $\xi$ in the form

$$
\xi=e^{-\lambda u} \zeta_{j}^{0}(\lambda)
$$

where $\zeta_{j}^{0}(\lambda)=0$ for $\lambda>0$ (it follows from the boundary condition), note that there are more conditions on the boundary values $\zeta_{j}^{0}(\lambda)$ in fact, being in $L^{2}$ 
just means

$$
\sum_{j} \int_{0}^{1} \int_{-\infty}^{0} e^{-2 \lambda u}\left|\zeta_{j}^{0}(\lambda)\right|^{2} d \mu_{j}(\lambda) d u(\text { Lebesgue })<\infty .
$$

Moreover we know that the boundary values are smooth. Then we simply define the continuous trace map

$$
\xi \longrightarrow\left(\chi_{(-\infty, 0)} \zeta_{j}^{0}(\lambda)\right)_{j}
$$

Denoting by $\mathcal{H}^{+}$the closure of the range of $\gamma^{+}$then $\gamma^{+}$becomes an essential isomorphism of $\Gamma$ Hilbert modules, so

$$
\operatorname{dim}_{\Gamma} \operatorname{Ker}\left(D^{+}\right)=\operatorname{dim}_{\Gamma} \mathcal{H}^{+} .
$$

This is a well known fact about Hilbert $\Gamma$ modules: if $f$ is an essential isomorphism (i.e. injective with range dense) then the isometry appearing in the polar decomposition of $f$ preserves the $\Gamma$ dimension of the modules. Doing the same for the kernel of $D^{-}$defines $\gamma^{-}$and $\mathcal{H}^{-}$and

$$
\operatorname{dim}_{\Gamma} \operatorname{Ker}\left(D^{-}\right)=\operatorname{dim}_{\Gamma} \mathcal{H}^{-} .
$$

So we have reduced the Ramachandran index to a boundary computation. Note that $\mathcal{H}^{+}$and $\mathcal{H}^{-}$are ortoghonal as happens in the classical theory. We can call $\mathcal{H}^{+} \oplus^{\perp} \mathcal{H}^{-}$the space boundary values.

REMARK 7.1. Everything works in exactly the same way for the Ramachandran index for a foliation transverse to the boundary equipped with a holonomy invariant transverse measure $v$. In this case one obtains a Borel field of boundary values whose $v$ dimension equals the dimension of the kernel of the operator. We briefly describe the situation. Let $X$ a compact manifold with boundary endowed with an even dimensional foliation $\mathcal{F}$ which is transverse to the boundary. Assume that the foliation is given with all the geometric datas needed to define a longitudinal generalized Dirac operator $D^{\mathcal{F}}$ acting on a leafwise Clifford bundle $S$. In [11] it is shown how to setup a leafwise generalized APS boundary value problem by imposing a Borel field of selfadjoint APS conditions. The realization of the corresponding operator is then Breuer-Fredholm in the von Neumann algebra of the foliation and an index formula is given. The trace map (7.3) can be defined leafwise for every leaf of the foliation with values on the spaces of section of the bundle on the foliation induced on the boundary. The von Neumann algebra of the foliation on the boundary embeds as a corner in the von Neumann algebra of the whole foliation hence the trace can be 
computed by a boundary computation measuring the $v$-dimensions of the spaces of the cauchy datas again.

Acknowledgments. We thank Paolo Piazza, Thomas Schick and Domenico Fiorenza for having suggested an investigation of the Ramachandran index for Galois coverings and foliations and for interesting discussions on the subject. The author wishes to thank the C.I.R.M of Trento where the paper was written.

\section{REFERENCES}

[1] M. F. Atiyah, V. K. Patodi and I. M. Singer. Spectral asymmetry and Riemannian geometry. I. Math. Proc. Cambridge Philos. Soc. 77 (1975), pp. 43-69.

[2] Paolo Antonini, The Atiyah Patodi Singer signature formula for measured foliations, http://arxiv.org/abs/0901.0143

[3] Paolo Antonini, The Atiyah Patodi Singer index formula for measured foliations, http://arxiv.org/abs/0907.0801. Accepted for publication on the Bull. Sci. Math.

[4] B. Boos-Bavnbeck, K. P. Wojciechnowski, Elliptic boundary value problems for Dirac operators. Birkhauser Boston, 1993.

[5] Manfred Breuer, Fredholm theories on Von Neumann algebras I, Math. Ann. 178 (1968), pp. 243-254.

[6] Manfred Breuer, Fredholm theories on Von Neumann algebras II, Math. Ann. 180 (1969), pp. 313-325.

[7] WoLFANG LUCK, $L^{2}$ Invariants: Theory and applications to geometry and $K-$ Theory, A Series of Modern Surveys in Mathematics, 44. Springer, Berlin, 2002.

[8] A. S. Mishchenko, A. T. Fomenko, The index of elliptic operators over $C^{*}$ algebras AT. Izv. Math. 15 (1980), pp. 87-112.

[9] J. RoE, An index theorem on open manifolds I, J. Differential Geom. 27 (1988), pp. 87-113.

[10] T. Schick, $L^{2}$-index theorems KK-Theory and connections, New York J. Math. 11 (2005), pp. 387-443.

[11] Mohan Ramachandran, Von Neumann index theorems for manifolds with boundary. J. Differential Geom. 38 (1993), pp. 315-349.

[12] JoHN ROE, Elliptic operators, topology and asymptotic methods, Pitman Research Notes in Mathematics Series, 395. Longman, Harlow, 1998.

[13] Michael Shubin, $L^{2}$ Riemann-Roch Theorem for Elliptic Operators, Geom. Func. Anal. 5 (1995), pp. 482-527.

[14] S. VASSOUT, Unbounded pseudodifferential calculus for Lie groupoids, J. Funct. Anal. 236 (2006), pp. 161-200.

[15] Boris VAillant, Index theory for coverings, http://arxiv.org/abs/0806.4043

Manoscritto pervenuto in redazione il 20 Marzo 2012. 